\title{
Dependence of the spike-triggered average voltage on membrane response properties
}

\author{
Laurent Badel, Wulfram Gerstner, Magnus J.E. Richardson* \\ École Polytechnique Fédérale de Lausanne (EPFL), Laboratory of Computational Neuroscience, School of Computer and Communication Sciences and Brain \\ Mind Institute, CH-1015 Lausanne, Switzerland
}

Available online 9 February 2006

\begin{abstract}
The spike-triggered average voltage (STV) is an experimentally measurable quantity that is determined by both the membrane response properties and the statistics of the synaptic drive. Here, the form of the STV is modelled for neurons with three distinct types of subthreshold dynamics; passive decay, h-current sag, and damped oscillations. Analytical expressions for the STV are first obtained in the low-noise limit, identifying how the subthreshold dynamics of the cell affect its form. A second result is then derived that captures the power-law behaviour of the STV near the spike threshold.
\end{abstract}

(C) 2006 Elsevier B.V. All rights reserved.

Keywords: Spike-triggered voltage; h-current; Damped voltage oscillations

\section{Introduction}

Reverse-correlation methods are a standard tool in the neurosciences [2]. The spike-triggered average voltage (STV) is a quantity that contains information about both the statistics of the synaptic drive and the response properties of the neuron, and can be readily extracted from intracellular voltage traces. An analytical understanding of the processes that shape the STV can be used to classify the response characteristics of neurons and the synaptic drive to which they are subjected [1].

Typically, the neuronal models that have been used to investigate the STV are of the integrate-and-fire (IF) type with leaky, passive dynamics $[1,8]$. The IF model, however, misses many important features of biological neurons such as the h-current sag seen in cortical and hippocampal pyramidal cells, as well as more complex behaviour such as damped oscillations seen in certain entorhinal cortical cells [4].

Here the form of the spike-triggered average will be investigated for model neurons with biologically more realistic dynamics. Two methods will be used: the first involves a low-noise approximation, which, in the context

\footnotetext{
*Corresponding author.

E-mail address: magnus.richardson@epfl.ch (M.J.E. Richardson).
}

of spiking neurons, corresponds to low-noise, noise-driven spike generation; the second method captures the powerlaw behaviour of the STV near threshold - a feature missed by low-noise approximations.

\section{The model}

The subthreshold voltage dynamics are described by a two-variable IF model $[3,5,9,10]$ which is sufficient to capture the response of neurons with passive dynamics, hcurrent sag response and damped oscillations. The model comprises a variable $v$ for the membrane voltage, with time-scale $\tau_{v}$ and a second variable $w$ proportional to the excess current flowing through voltage-gated trans-membrane channels, with time-scale $\tau_{w}$ and coupled with strength $\gamma$ to the voltage. Thus,

$\tau_{v} \dot{v}=\mu-v-\gamma w+\sqrt{\tau_{v}} \sigma \xi(t)$ and $\tau_{w} \dot{w}=v-w$.

The variable $\mu /(1+\gamma)$ is the drive-dependent equilibrium potential and $\xi(t)$ is delta-correlated white noise multiplied by an amplitude factor $\sigma$. The subthreshold dynamics are supplemented by a voltage threshold $v_{\text {th }}=-55 \mathrm{mV}$ and reset $v_{\text {re }}=-65 \mathrm{mV}$ that provide the spike mechanism. The standard integrate-and-fire dynamics are recovered when $\gamma=0$. 


\section{Spike-triggered average voltage}

\subsection{Low-noise approximation}

Our calculation is based on a principle which states that for a set of equations of the type (1), the most likely trajectory followed by the system when moving from one point to another is obtained by maximising the probability of the synaptic input $\sqrt{\tau_{v}} \sigma \xi(t)$ that would yield such a transition. This optimisation is carried through the minimisation of a cost function that will now be derived (the informal derivation given here follows the well-known principle of minimum available noise energy [7]).

We start by introducing a time-discretised version of the system (1)

$\tau_{v}\left(v_{k-1}-v_{k}\right)=-\left(v_{k}+\gamma w_{k}\right) \Delta+\sqrt{\sigma^{2} \tau_{v} \Delta} \Psi_{k}$,

$\tau_{w}\left(w_{k-1}-w_{k}\right)=\left(v_{k}-w_{k}\right) \Delta$,

where $\Psi_{k}$ are independent Gaussian random numbers with zero mean and unit variance, $\Delta$ is a small time interval, and the variables have been rescaled so that the equilibrium point is $(v, w)=(0,0)$. It should be noted that the label $k$ counts backwards in time from the threshold crossing. It is given that the neuron is at rest at time $T=-n \Delta$ (where $n$ is the number of discrete time bins) and at threshold at time 0 . The probability for the voltage to follow a particular trajectory between these two points is proportional to the probability of the sequence of random numbers $\Psi_{k}$ that would yield this trajectory,

$$
\begin{aligned}
& p\left(v_{n}, w_{n},-n \Delta ; \ldots ; v_{0}, w_{0}, 0\right) \\
& \quad \propto \prod_{k=1}^{n} \exp \left(-\frac{1}{2 \sigma^{2} \tau_{v}}\left[\tau_{v} \frac{v_{k-1}-v_{k}}{\Delta}+\left(v_{k}+\gamma w_{k}\right)\right]^{2} \Delta\right),
\end{aligned}
$$

where $v_{n}, w_{n}=0$ (the neuron is at rest) and $v_{0}=v_{\text {th }}$ (a spike is emitted). This equation is supplemented by the constraint that the deterministic equation for the second variable $w$ should be satisfied. When the continuum limit is taken, this expression becomes

$p(v(t), w(t)) \propto \exp \left(-\frac{1}{2 \sigma^{2} \tau_{v}} \int_{T}^{0}\left(\tau_{v} \dot{v}+v+\gamma w\right)^{2} \mathrm{~d} t\right)$.

From this, we see that in the limit of vanishing noise $(\sigma \rightarrow 0)$, the trajectory of highest probability which dominates the dynamics is that which minimises the integral (under the constraint that $v=\tau_{w} \dot{w}+w$ ). It is this trajectory, analogous to the maximum-likelihood escape trajectory [8] identified for the one-variable IF model, that will be derived. It should also be noted that taking $T \rightarrow$ $-\infty$ yields a scenario in which the neuronal voltage approaches the threshold from its equilibrium distribution: this limit is taken.

If we insert the $v$ constraint into the above integral, we obtain a variational problem for $w$ in which the functional

$\mathscr{S}(w(t))=\int_{-\infty}^{0}\left(\tau_{v} \tau_{w} \ddot{w}+\left(\tau_{v}+\tau_{w}\right) \dot{w}+(1+\gamma) w\right)^{2} \mathrm{~d} t$

must be minimised over all possible trajectories $w(t)$ starting from 0 at time $-\infty$ and ending at an arbitrary point $w(0)$ at time 0 . This point will be determined later. Solving the Euler-Lagrange equation for this problem leads to $w(t)=A \mathrm{e}^{-\lambda_{1} t}+B \mathrm{e}^{-\lambda_{2} t}$, where $\lambda_{1,2}$ are the eigenvalues associated with the equation set (1). $A$ and $B$ are integration constants; one of them is determined by the condition $v(0)=v_{\text {th }}$, while the other is obtained by further minimising the functional over all possible values of $w(0)$. The voltage trajectory is then calculated from $v=\tau_{w} \dot{w}+w$, yielding

$v(t)=\frac{v_{\text {th }}}{\lambda_{1} \lambda_{2} \tau_{w}^{2}+1}\left(\frac{\lambda_{2}\left(\lambda_{1}^{2} \tau_{w}^{2}-1\right)}{\lambda_{1}-\lambda_{2}} \mathrm{e}^{-\lambda_{1} t}+\frac{\lambda_{1}\left(\lambda_{2}^{2} \tau_{w}^{2}-1\right)}{\lambda_{2}-\lambda_{1}} \mathrm{e}^{-\lambda_{2} t}\right)$.

The resulting trajectories for three examples (passive decay, h-current sag and damped oscillations) are shown in Fig. 1. For the passive IF case, the low-noise approximation is equivalent to the time-reversed relaxation trajectory result

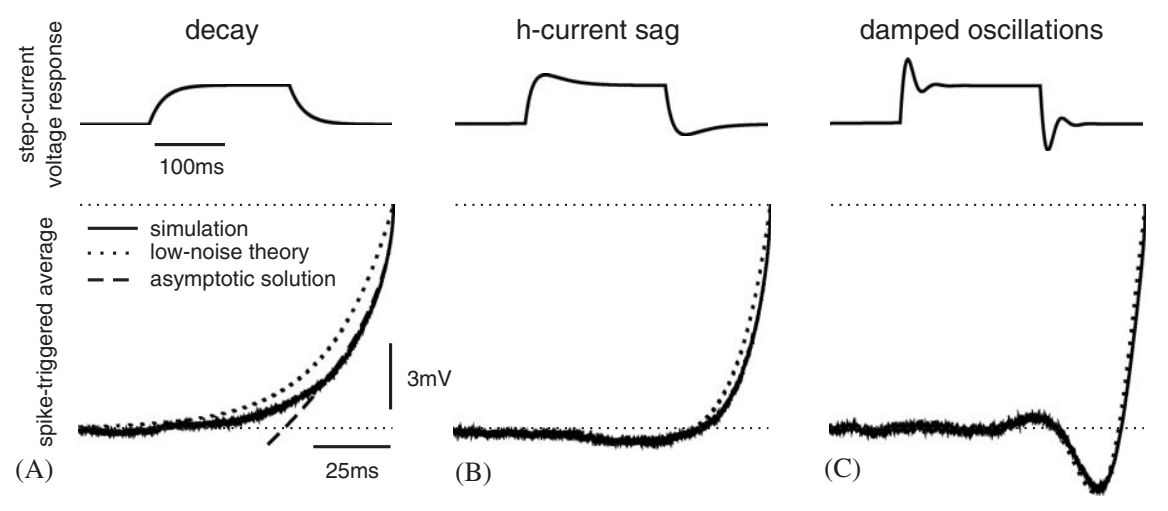

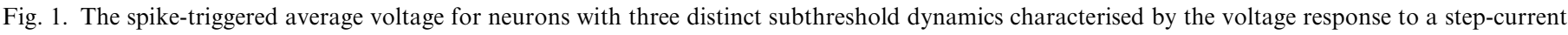

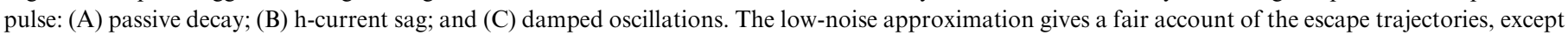

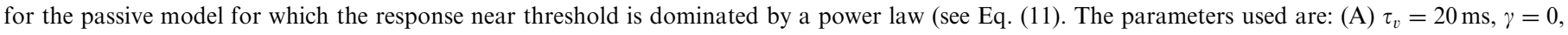

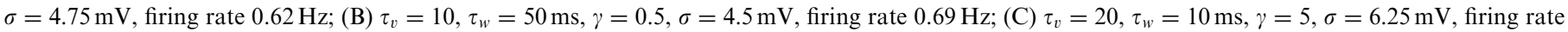
$0.50 \mathrm{~Hz}$. In all cases $\mu /(1+\gamma)=v_{\text {re }}$. 
[6]. From Fig. 1B, the case of an h-current, it can be seen that given a spike it was likely that there was a small amount of early inhibition: this has the effect of switching on the h-current which helps to depolarise the neuron. Similar, more pronounced behaviour is seen for the case of a neuron with damped oscillations in Fig. 1C.

\subsection{Behaviour near the threshold}

The low-noise approximation is inaccurate near threshold as can be seen in Fig. 1A. However, an (asymptotically) exact form can be derived for the region near threshold under the assumption that noise dominates over the $w$ and $v$ leak currents. The first step is to expand the conditional voltage distribution using Bayes's rule as

$p(v,-t \mid$ spike at $t=0)=\frac{\varphi(v)}{r} \rho(v,-t)$,

where $\varphi(v)=p(v,-t)$ is the stationary distribution of voltage, and $r$ is the spike rate. $\rho$ is the conditional spike rate at time 0 given the value $v$ of the voltage at some earlier time $-t$. Since we are interested in the behaviour near the threshold, i.e., at small times before the spike, this quantity is well approximated by the first-passage-time density to the threshold, which we denote by $\rho_{1}(v,-t)$. If we assume that noise dominates over the leak currents, the latter quantity can be calculated using standard methods:

$\rho_{1}(v,-t)=\frac{1}{t} \frac{\left(v_{\mathrm{th}}-v\right)}{\sqrt{2 \pi \sigma^{2} t / \tau_{v}}} \exp \left(-\frac{\left[v_{\mathrm{th}}-v\right]^{2}}{2 \sigma^{2} t / \tau_{v}}\right)$.

By considering the probability current, the distribution $\varphi(v)$ near the threshold can be found to be

$\varphi(v)=\frac{2 \tau_{v} r}{\sigma}\left(\frac{v_{\mathrm{th}}-v}{\sigma}\right)+O\left[\left(\frac{v_{\mathrm{th}}-v}{\sigma}\right)^{2}\right]$.

Using approximations (8) and (9) in (7), we can write the STV as

$v_{\text {th }}-v_{\text {stv }}(t) \simeq \int_{-\infty}^{v_{\text {th }}}\left(v_{\text {th }}-v\right) \frac{\varphi(v)}{r} \rho_{1}(v,-t) \mathrm{d} v$

and obtain the asymptotic form

$v_{\mathrm{stv}}(t) \simeq v_{\mathrm{th}}-\sigma \sqrt{\frac{8 t}{\pi \tau_{v}}}$.

This is also plotted in Fig. 1A and can be seen to give a satisfactory account of the run-up to the spike. It should be noted that noise always dominates over the deterministic terms sufficiently close to threshold, regardless of the exact form of the leak currents. The boundary layers are, however, much smaller for the h-current sag and damped oscillations models and therefore not included in Figs. 1B and $\mathrm{C}$. For cases of stronger noise the boundary layer exhibiting the power-law decay will penetrate further into the subthreshold domain.

\section{Discussion}

We have derived analytical expressions for the spiketriggered average voltage (STV) of three model neurons with subthreshold dynamics corresponding to those seen in biological neurons: passive decay, h-current sag and damped oscillations. Our analyses demonstrate that (i) the form of the STV is closely related to the subthreshold membrane dynamics, and (ii) for a hard-threshold model with white noise the STV behaves as a $\sqrt{t}$ power-law near threshold. The distinct forms for the STV derived here can be included in models of spike-timing-dependent synaptic plasticity to investigate the effects of subthreshold membrane properties on the dynamics of the synaptic weights.

Although fixed-threshold models (such as the generalised IF models here) are commonly used, they do not provide a good model for spike generation. Other, non-linear, models with soft thresholds (such as those containing the full sodium and potassium spike-generating currents) are more realistic. Since the subthreshold behaviour is similar for both types of models away from threshold, part (i) of our results is relevant to the analysis of the voltage traces of biological neurons. Part (ii) of our results, however, is rather of technical interest to theoreticians working with IF neurons.

Here, we examined the influence of membrane properties on the STV. The rich statistics of biological synaptic drive, here simplified to Gaussian white noise, are also likely to affect the form of the STV. The influence of temporal correlations and conductance effects in the synaptic drive, and non-linearities in the voltage-activated currents provide topics for further study. However, a preliminary analysis did not show qualitative differences with the results presented for the simplified model here.

\section{Acknowledgements}

We thank Liam Paninski for useful discussions. This work was supported by Grant no. 200020-108093/1 of the Swiss National Science Foundation.

\section{References}

[1] B. Agüera y Arcas, A. Fairhall, What causes a neuron to spike?, Neural Comput. 15 (2003) 1789-1807.

[2] F. de Boer, P. Kuyper, Triggered correlation, IEEE Trans. Biomed. Eng. 15 (1968) 169-179.

[3] N. Brunel, V. Hakim, M.J.E. Richardson, Firing rate resonance in a generalized integrate-and-fire neuron with subthreshold resonance, Phys. Rev. E 67 (2003) 051916.

[4] E. Fransén, A.A. Alonso, C.T. Dickson, J. Magistretti, M.E. Hasselmo, Ionic mechanisms in the generation of subthreshold oscillations and action potential clustering in entorhinal layer II stellate neurons, Hippocampus 14 (2004) 368-384.

[5] E.M. Izhikevich, Resonate-and-fire neurons, Neural Networks 14 (2001) 883-894.

[6] J. Kanev, G. Wenning, K. Obermayer, Approximating the responsestimulus correlation for the integrate-and-fire neuron, Neurocomput. 58-60 (2004) 47-52. 
[7] R.L. Kautz, Thermally induced escape: the principle of minimum available noise energy, Phys. Rev. A 38 (1988) 2066-2080.

[8] L. Paninski, The most likely voltage path and large deviations approximations for integrate-and-fire neurons, J. Comput. Neurosci. (2006), to appear.

[9] M.J.E. Richardson, N. Brunel, V. Hakim, From subthreshold to firing-rate resonance, J. Neurophysiol. 89 (2003) 2538-2554.

[10] A. Tonnelier, W. Gerstner, Piecewise linear differential equations and integrate-and-fire neurons: insights from two-dimensional membrane models, Phys. Rev. E 67 (2003) 021908.

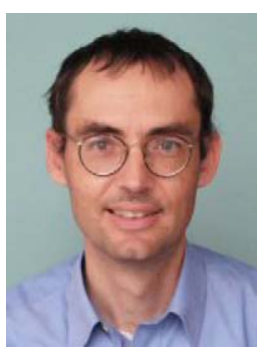

Wulfram Gerstner received his Ph.D. degree in theoretical physics from the TU Munich, Germany, in 1993, after studies in Tübingen, Berkeley, and Munich. He is a Professor and Head of the Laboratory of Computational Neuroscience, EPFL, Switzerland.

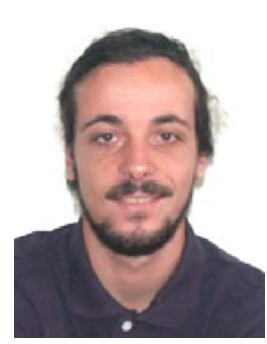

Laurent Badel is a Ph.D. student at the Laboratory of Computational Neuroscience (LCN) at the EPFL. He received his M.Sc. in physics from the EPFL in march 2003. His current research interests are mathematical and statistical approaches to the analysis of neuronal systems.

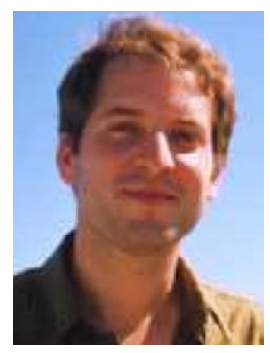

Magnus Richardson obtained his D-Phil in theoretical physics in 1997 at the University of Oxford. Since then he has worked on various subjects in the neurosciences and is currently interested in how neuronal properties lead to emergent states at the level of neural networks. 